\title{
Habilidades socioafectivas para la solución pacífica de conflictos: caso Malala Academia, A.C.
}

\author{
Patricia Moya Grijalva y Jacobet Rosas Yépez*
}

\section{Resumen}

El presente trabajo es producto de la experiencia de la implementación del curso taller La afectividad y creatividad en la gestión pacífica de conflictos, dirigido a adolescentes de doce a dieciséis años que asisten a Malala Academia, A.C. en la colonia San Luis, ubicada al oriente de la ciudad de Hermosillo, Sonora. Este fue un proyecto de vinculación con la Universidad de Sonora. Estuvo enfocado principalmente en brindar herramientas que favorecen la resolución de conflictos de una manera creativa y pacífica, y a la vez, coadyuvar para que los menores construyan relaciones asertivas. Así mismo, se trabajó el potencial afectivo con el propósito de que los participantes elevaran su autoestima y aprendieran a manejar asertivamente sus sentimientos, emociones y autonomía. El desarrollo de las actividades se fundamentó en el modelo cognitivo conductual. Durante las sesiones de trabajo se buscó que el participante obtuviera elementos que le permitieran identificar sus propios patrones de conductas no asertivas, reflexionar y modificarlos, lo cual tendrá un impacto propositivo en la forma de actuar ante la presencia de un conflicto. Se utilizaron técnicas de participación activa, de integración, participación grupal, de análisis, de reflexión y análisis de casos y audiovisuales. La actividad se llevó acabo de agosto a diciembre de 2017 y se planea continuar con el fomento al desarrollo de las habilidades para la construcción de paz en los mismos menores.

\footnotetext{
* Maestras de tiempo completo de la Licenciatura en Trabajo Social de la Universidad de Sonora. Correos electrónicos: patricia@sociales.uson.mx y jrosas@sociales.uson.mx
} 


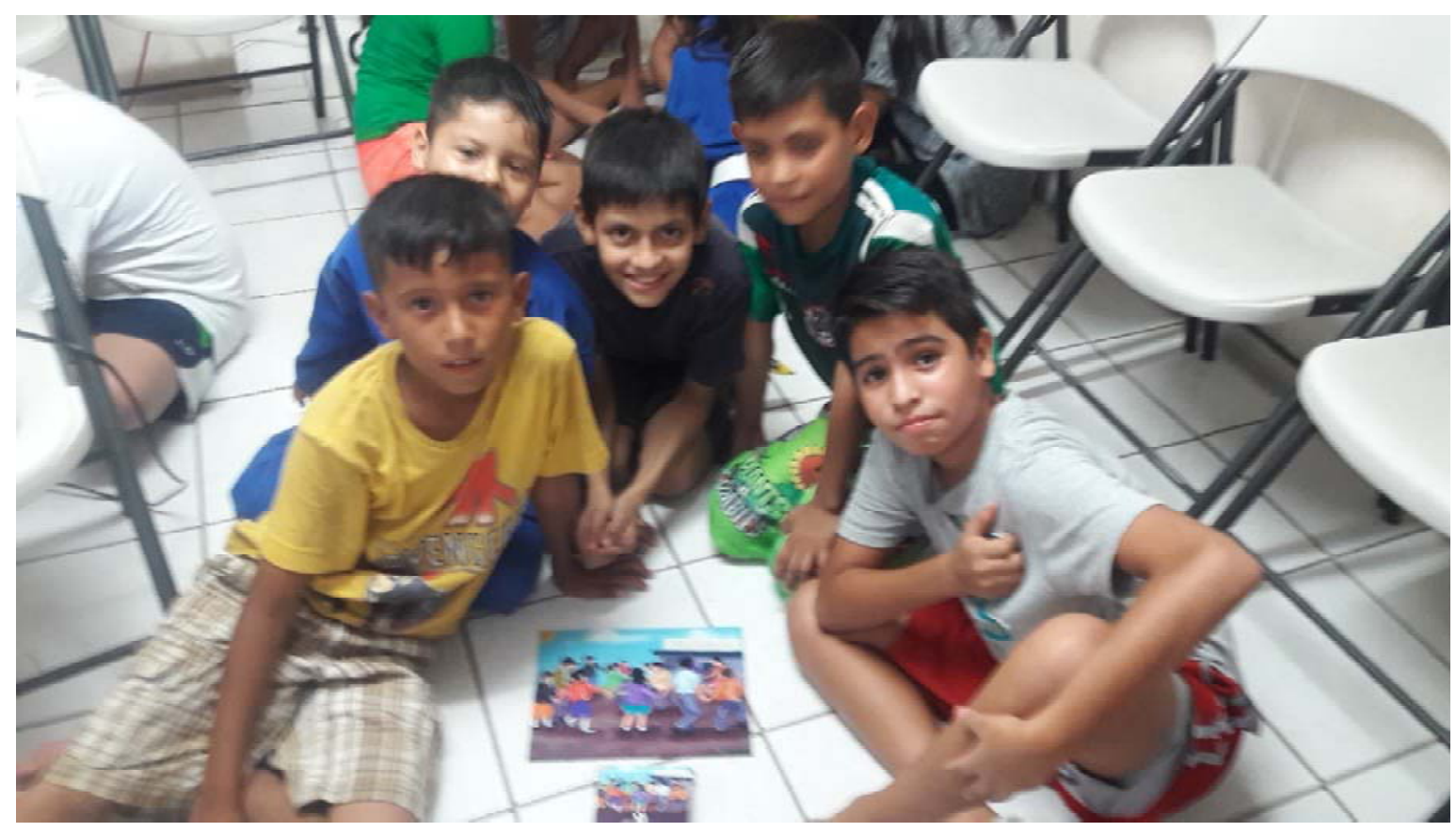

Foto: Patricia Moya Grijalva. Sesión de trabajo.

\section{Justificación}

La comunicación inadecuada, las fallas en la toma de decisiones, las relaciones interpersonales y familiares no asertivas, tienen efectos negativos en las personas; si a esto se suma el que todos los seres humanos son y piensan diferente, es natural que se presenten situaciones conflictivas a lo largo de la vida, de las que nadie está exento. Los conflictos son inevitables, solo que deben afrontarse de manera efectiva.

La colonia San Luis ubicada al oriente de la ciudad de Hermosillo, Sonora, está considerada como una de las colonias más vulnerables, ya que se presentan problemas de pandillerismo, delincuencia y adicciones; aunado a ello, personas en condición de indigencia o migrantes en tránsito merodean la colonia debido a la cercanía con la estación de ferrocarril $y$ que en algunos casos provocan sentimientos de inseguridad en la población.

La intervención en este curso taller se deriva de la vinculación social que se estableció entre la Universidad de Sonora y Malala Academia, A.C., con el propósito de contribuir en la integralidad de sus programas y coadyuvar con la institución que promueve la educación y valores en los niños y jóvenes en la mencionada colonia, para que los menores de doce a dieciséis años, en riesgo de incurrir en adicciones, delincuencia, deserción escolar y/o violencia, potencien las habilidades que favorezcan la resolución pacífica de conflictos y la toma adecuada de decisiones.

De acuerdo con la Organización Mundial de la Salud, la adolescencia es un lapso de edad que va de los diez a los veinte años, con variaciones culturales $e$ 
individuales. Gallego (2006), nos señala que:

Los cambios de la adolescencia suelen comenzar antes que los de la pubertad y continúan después: son unos cambios que no se refieren solamente al cuerpo del adolescente, sino a modo de existir de su persona. Es una etapa con un gran desarrollo biológico social, emocional y cognitivo. La adolescencia es un periodo de transición entre dos momentos evolutivos; la infancia y la edad madura.

Es una etapa con grandes variaciones en los deseos y aspiraciones. En ella manifiestan deseos de conocimientos concretos, de aceptación social, de poseer un cuerpo bello, de tener dinero, de poseer al otro sexo, de sueños imposibles, de aspiraciones desmedidas. Con continuas variaciones en los estados de ánimo: alegría y nostalgia, entusiasmo y depresión o angustia, inestabilidad emocional, rebeldía rabietas y sentimientos de incomprensión son otros acompañantes en este estadio de la vida, en la que además uno cree que ya lo sabe todo y lo expresa apasionadamente.

Es importante destacar que los seres humanos no son seres aislados y que por lo tanto, deben adquirir conciencia de sí mismos y de la sociedad que les rodea. Esto conlleva al desarrollo humano, que es concebido por Loaiza, Salazar, Santacoloma y León (2015) como: "Un proceso a través del cual una persona puede llegar efectivamente a constituirse como tal, tanto en los aspectos que lo diferencian de los demás y lo hacen singular, como en aquellos que le permiten ser miembro de los colectivos, lo cual le implica su posicionamiento histórico y social".

Siguiendo con estos autores, en el desarrollo humano están inmersas las siguientes esferas:

1. La esfera orgánica madurativa, en la cual se identifican las condiciones biológicas, físicas y neurológicas que le sirven de soporte a las demás esferas del desarrollo humano.

2. La esfera cognitiva, que puede ser reconocida por cuanto permite la posibilidad de realizar representaciones del mundo, solucionar creativamente los problemas, efectuar aprendizajes de orden intelectual, comprender las diversas formas de simbolización y de códigos comunicativos.

3. La esfera ético moral, relacionada con la construcción de posibilidades para la interacción y la convivencia, la generación de unos mínimos que permitan el reconocimiento del otro, de los otros, la verosimilitud de conductas autónomas y el asumir responsablemente las consecuencias de los actos libremente asumidos.

4. La esfera lúdica, en la cual la persona reconoce y ejerce su libertad que a su vez le permitirá no sólo expresarse plenamente, sino crear nuevas condiciones. Es la lúdica un acto vital que trasciende el propio juego, en tanto permite explorar, transformar $y$ pensar.

5. La esfera erótico afectiva, a través de la cual se establecen las relaciones vinculantes de orden afectivo, consigo mismo (construcción de la identidad, el sentimiento de sí mismo) y con los demás (la vida de pareja, la integración a una familia o a diversos grupos filiales 0 simplemente cercanos).

6. La esfera productiva laboral, en la cual se planifica el trabajo como producción humana y como posibilidad de generación de nuevas alternativas y posibilidades.

7. La esfera política, relacionada con la generación de formas de organización de la vida pública, la participación, la búsqueda del bien común y la creación de condiciones de posibilidad de constituirse como miembro maduro de un estado. 
8. La esfera social, gracias a la cual se da la posibilidad de interactuar y dar sentido y significado a los productos de tal interacción.

9. La esfera comunicativa, relacionada con el desarrollo del lenguaje y la comunicación tanto posibilidad de diálogo, de argumentación, dentro de la racionalidad comunicativa.

Estas esferas que constituyen al ser humano, no se presentan de manera simultánea, sino en diferentes ritmos y etapas del ciclo vital; durante dicha transición, los afectos son muy importantes, ya que contribuyen fundamentalmente en niños $y$ adolescentes, a propiciar seguridad, madurez, a construir la identidad y a lograr una personalidad equilibrada.

La afectividad es un elemento que contribuye a la adaptación del sujeto, guía sus acciones y reacciones e impacta en sus relaciones; engloba todos los estados emocionales que se arraigan en el inconsciente $y$ es fundamental en el desarrollo humano. De acuerdo con Botia, et al. (2007), la afectividad es:

Una capacidad propia de toda persona de tener relaciones positivas con ella misma, con las demás personas, con la naturaleza y el entorno en general. Es decir, es una capacidad humana básica y fundamental para el desarrollo de la persona, algo estructural en el ser humano.

De hecho, las relaciones afectivas abarcan un amplio abanico de posibilidades:

$>$ El amor de una persona a sí misma, a su cuerpo, a sus proyectos, a su vida.

$>$ Las relaciones de simpatía, solidaridad y amistad con las personas
$>$ Las relaciones de filiación, maternidad, paternidad, fraternidad, solidaridad

- Las relaciones con la naturaleza, con los animales

$>$ Las relaciones de positividad con los objetos, con el hábitat natural y urbano

> La participación en la construcción social desde criterios éticos.

Loaiza et al. (2015) mencionan que: "La dimensión subjetiva en la afectividad parte de una idea general de 'autorreconocimiento' entendida como la conciencia que se tiene de lo que se es y lo que se puede lograr. Para ello es importante desarrollar el cuidado de sí, el auto concepto (autoimagen y autoestima) y la identificación y expresión de sentimientos y emociones".

El cuidado de sí representa el desaprender aquellas formas de pensar y actuar que fueron aprendidas y a partir de la relación consigo mismo, es necesario modificar, darles un nuevo significado y reaprender nuevos discursos y prácticas, de acuerdo con el ser humano que se desea ser; en cuanto al autoconcepto, es el reconocimiento e imagen que cada persona tiene de sí, de sus capacidades, cualidades y características. La identificación de sentimientos y emociones se relaciona con el potencial que tiene la persona para comprender y expresar sus sentimientos y emociones; pero además, reconocer aquellas situaciones o personas que le generan cierto tipo de emociones que le llevan a expresar de forma particular un pensamiento o un sentimiento.

Además, se debe considerar a la afectividad como un elemento clave en las relaciones humanas, ya que en ella se involucran los sentimientos y emociones. Es importante también tener en cuenta las capacidades comunicativas y empáticas 
que permiten relaciones interpersonales más sanas y al comprender los sentimientos propios y los de los demás, potencia las posibilidades de generar y elegir alternativas creativas para la solución pacífica de los conflictos.

La creatividad, según García García (1998): "puede ser catalogada como un proceso, es decir, como un conjunto de etapas que se suceden desde antes de la generación de una idea hasta el reconocimiento y elaboración final de la misma. Las etapas que conforman el proceso creativo son las siguientes: encuentro con el problema, generación de ideas, elaboración de la idea y transferencia creativa".

Los potenciales afectivo y creativo, se van desarrollando a lo largo de la vida del ser humano y se debe poner especial atención en el ejercicio de las capacidades comunicativas y empáticas; en esta experiencia, se atendió a adolescentes, ya que se considera que esta es una etapa de cambios físicos, psicológicos, emocionales y sociales que se generan de manera dinámica, por lo que es necesario que cuenten con información y herramientas que contribuyan a fortalecer su resiliencia, comunicación asertiva, autoestima y en general, su desarrollo humano.

Los citados potenciales favorecen la actuación del ser humano al enfrentar un conflicto -el cual es inherente a la naturaleza del ser mismo-, y para evitar que prospere es necesario afrontarlo, a fin de resolverlo preferentemente, en el momento en que se presenta; de lo contrario, el conflicto crecerá afectando a los involucrados y puede impactar también en terceras personas.

En ocasiones, se presentan problemas que emergen de conflictos que no fueron resueltos con oportunidad o de manera satisfactoria.
De acuerdo con Pérez y Pérez (2011), el conflicto se produce cuando existe un desacuerdo o diferencia de opiniones, intereses 0 necesidades entre las personas. El desacuerdo puede variar en intensidad, según la postura de los implicados.

Los conflictos pueden ser de dos tipos: intrapersonal, cuando se genera al interior del sujeto mismo producto de sus insatisfacciones $\mathrm{o}$ problemas; $\mathrm{e}$ interpersonal, cuando es producto de diferencias con otros.

Para dirimir un conflicto, un método muy antiguo que en la actualidad ha tomado fuerza y se ha perfeccionado es el de la mediación. Pérez Serrano (2011), menciona que es la "forma pacífica de resolver conflictos entre dos 0 más personas con la ayuda de una tercera, el mediador que ha de ser imparcial y neutral, precisa la responsabilidad de los participantes en la toma de decisiones, facilita la comprensión de las causas del conflicto y lo hace más manejable cuando no puede ser solucionado totalmente".

En virtud de lo anterior y como el adolescente se encuentra en una etapa de desarrollo formativa y la institución en la que se desarrolló el curso taller está inserta en una colonia considerada conflictiva, del análisis de un abanico de posibles alternativas de intervención, se juzgó prioritario promover una cultura de la paz a través del curso taller $\mathrm{La}$ afectividad y creatividad en la gestión pacífica de conflictos.

\section{Objetivo general de la intervención}

Brindar a los adolescentes que asisten a Malala Academia, A.C., herramientas que contribuyan al desarrollo de habilidades que favorezcan la resolución pacífica de conflictos a través de talleres lúdico educativos. 


\section{Objetivos específicos}

- Ofrecer herramientas de disciplina positiva para potenciar el autocontrol emocional y la conciencia social.

- Ofrecer herramientas de análisis para favorecer la toma adecuada de decisiones.

- Sensibilizar sobre la importancia de actuar con conciencia social para comprender mejor a los otros.

- Fomentar el pensamiento creativo para la resolución de situaciones de peligro o conflicto.

\section{Contextualización y reconstrucción de la práctica}

Malala Academia es una asociación civil sin fines de lucro, que como institución educativa tiene entre sus objetivos brindar servicios educativos y deportivos a niños $y$ jóvenes de escasos recursos y con alta vulnerabilidad, brindando el soporte y los valores necesarios para que puedan mejorar su calidad de vida. Esta institución utiliza un modelo donde por cada hora de estudio/tarea, los alumnos tienen derecho a una hora de entrenamiento deportivo. De tal forma que, en la academia, los alumnos del programa de permanencia escolar Malala, no cubren su estancia con dinero, sino con esfuerzo, disciplina y estudio.

Los orígenes de esta institución son de Culiacán, Sinaloa e inició actividades en nuestra ciudad en febrero de 2016, sita en la colonia San Luis, con el propósito de fomentar en niños y jóvenes la ética del cuidado, valores y trabajo a través de un programa integral de apoyo social, a fin de evitar la deserción escolar, prevenir adicciones y el delito.

La intervención en el curso taller $\mathrm{La}$ afectividad y creatividad en la gestión pacífica de conflictos, se deriva de la vinculación social que se estableció entre la Universidad de Sonora y Malala Academia, A.C., con el propósito de contribuir en la integralidad de sus programas y coadyuvar con la institución, la cual promueve la educación y los valores en los niños y jóvenes en la mencionada colonia, para que los menores de doce a dieciséis años, en riesgo de incurrir en adicciones, delincuencia, deserción escolar y/o violencia, potencien las habilidades que favorezcan la resolución pacífica de conflictos y la toma adecuada de decisiones. La intervención fue dirigida por maestras y ejecutada con la participaron de dos estudiantes del sexto semestre de la Licenciatura en Trabajo Social de la Universidad de Sonora, que ejercitaron competencias de trabajo social con grupos.

\section{Descripción de la práctica}

El trabajo realizado en la institución del sector social que tiene entre sus propósitos brindar servicios educativos y deportivos a niños y jóvenes de escasos recursos y con alta vulnerabilidad, procurando el soporte y los valores necesarios para que puedan mejorar su calidad de vida, tuvo como base el pensar que lo afectivo es toral en la construcción de la ciudadanía y la paz y lo creativo para desarrollar y ampliar el espectro de alternativas para resolver conflictos y tomar decisiones. Sin embargo, durante el curso taller se dio una mayor inclinación al tema de la autonomía y a las habilidades de la mediación como herramienta de transformación de conflictos.

El desarrollo de las actividades se fundamenta teóricamente en el desarrollo humano y principalmente estuvo dirigido a 
desarrollar procesos de democracia y construcción de paz.

Así mismo, se utilizó el modelo cognitivo conductual, el cual, de acuerdo con Fernández y López (2006), se basa en dos principios básicos: uno hace referencia a que conductas, emociones y pensamientos son consecuencia del aprendizaje, por lo que pueden transformarse; y el otro, a la relación homogénea entre el pensamiento y la conducta; es decir, un cambio en las creencias o forma de interpretar los datos llevará a modificar la conducta.

Por lo anterior, con la utilización de este modelo se buscó reestructurar patrones cognitivos como forma de generar un cambio, tanto a nivel conductual como cognitivo.

Durante las sesiones de trabajo se buscó que el participante obtuviera elementos que le permitan modificar formas de pensar y como consecuencia, de actuar pro-positivamente ante la presencia de un conflicto. La propuesta de trabajo estuvo organizada en la modalidad de curso taller, por lo que se trabajó a través de la exposición de elementos teóricos que permitieron tener un punto de partida común, para posteriormente combinar la explicación y reflexión con ejercicios lúdicos y prácticos que modelaron lo revisado teóricamente. Se utilizaron técnicas de: participación activa, integración y participación grupal, análisis, reflexión y análisis de casos y audiovisuales. Las actividades se realizaron en sesiones semanales de dos horas cada una, durante tres meses.

Los contenidos que se abordaron fueron: autoestima, control emocional, comunicación, asertividad y escucha activa, conciencia social, mediación como herramienta de transformación de conflictos, creatividad para resolver conflictos, autonomía y valores para la convivencia.
Dichos temas permitieron a los menores: adquirir elementos para mejorar su autovaloración y conciencia de sí mismos; obtener herramientas que contribuyan a potenciar su control emocional y asertividad ante las diversas situaciones que se les presenten, en los diferentes ámbitos en los que se desenvuelven; tener una interacción más asertiva en sus contextos cotidianos; afrontar los conflictos con creatividad y hacer uso de estrategias de mediación para la resolución pacífica de los mismos; dotarse de herramientas para actuar con autonomía responsable y valores para la convivencia.

Al finalizar cada tema, se aplicaron instrumentos de evaluación, tanto para los participantes, como para las cuatro instructoras, cuyos resultados fueron satisfactorios. El $75 \%$ de los menores que participaron en el curso taller, ejercitaron acciones para un mayor autoconocimiento, lograron comunicarse con mayor asertividad, conocieron $y$ aplicaron el proceso para la toma de decisiones y técnicas de autocontrol emocional. Así mismo, obtuvieron herramientas que les permitirán enfrentar con creatividad situaciones de peligro o conflicto, y fueron sensibilizados para actuar con conciencia social. El $10 \%$ de los participantes no asistieron con regularidad al curso taller y de un $15 \%$ no se observaron cambios significativos en su comportamiento.

\section{Interpretación crítica}

Durante la experiencia, se advirtió un cambio progresivo en el comportamiento de la mayoría de los adolescentes, así como en las interrelaciones con sus compañeros y con las autoridades de la institución. El trabajo colaborativo, la participación y el entusiasmo de los 
adolescentes, permitió el logro de los objetivos. Cabe resaltar, que los participantes contribuyeron a la construcción de su aprendizaje y lo volvieron significativo.

La participación de los menores al inicio fue poca. Su comportamiento en el aula era hasta cierto punto indisciplinado. La mayoría se mantenía únicamente como receptores de la información, aunque participaban en las técnicas que se aplicaban; poco a poco fueron adquiriendo confianza y empezaron a tener una participación más activa y entusiasta, realizando los trabajos en equipo de manera colaborativa.

A lo largo del desarrollo del curso, las formas de comunicación entre los participantes tuvieron un cambio observable, ya que dejaron de gritarse para dirigirse al otro con respeto y de manera asertiva.

Sin embargo, de los veinte niños que participaron, el $25 \%$ no alcanzó los objetivos del curso.

\section{Conclusiones}

Este tipo de cursos-talleres, complementados con las actividades que desarrolla Malala Academia, A.C., se convierten en factores protectores para los niños y adolescentes que asisten a la institución, a la vez que contribuyen a su desarrollo humano armónico e integral, previenen la comisión de delitos.

Por ello, se tiene contemplado continuar trabajando con estos menores en otras esferas del desarrollo humano, a fin de potenciar sus habilidades, actitudes, valores y conocimientos que los lleven a sostener interrelaciones personales sanas, construir una cultura de la paz y una mejor sociedad.

\section{Prospectiva}

De acuerdo con los resultados obtenidos con la implementación del curso taller, se considera que este tipo de trabajo con los adolescentes es importante desarrollarlo también en otras instituciones sociales que busquen mejorar el desarrollo humano y social. Actualmente, el deterioro de los valores, las escasas habilidades sociales y el aumento de la presencia de conflictos interpersonales, hace necesario desarrollar, desde la niñez y adolescencia, alternativas creativas y pacíficas para la resolución de las diferencias, como un factor primario en busca de mejores escenarios de convivencia en la escuela, la familia y la comunidad.

Así mismo, se continuará trabajando en Malala, tanto con los menores, como con las madres de familia que llevan a sus hijos, a fin de que estas también desarrollen habilidades para la solución pacífica de conflictos y apoyen en el proceso de aprendizaje de sus hijos de una cultura para la paz.

\section{Bibliografía}

André, C. y Lelord, F. (2009). La Autoestima: gustarse a sí mismo para mejor vivir con los demás. ( $5^{\mathrm{a}}$ ed.). Barcelona: Kairós.

Botia, F., Catalán, E., Font, P., Grande, A., López Sánchez, F., Manzaneque, C., ... Vega, S. (2007). Sexualidad, identidad y afectividad. Cómo tratarlas desde la escuela. Barcelona: Laboratorio Educativo.

Cardona, A. (2000). Formación de valores: Teoría, reflexiones y respuestas. México: Grijalbo.

Fernández, T. y López, A. (2006). Trabajo social con grupos. Madrid: Alianza Editorial, S.A. 
Foderaro, A., Schmidt, D. (2012). Tu decisión para adolescentes. Guía de sentido común para tomar mejores decisiones. Bloomington, IN: Author House.

Gallego Codes, J. (2006). Educar en la Adolescencia. Madrid: Thomson Ediciones Paraninfo, S.A.

García García, J.J. (1998). La creatividad y resolución de problemas como base de un modelo didáctico alternativo. Educación y Pedagogía, (21), 145-173.

Goleman, D. (2008). Inteligencia Emocional. Barcelona: Kairós.

Güell Barceló, M. (2006). ¿Por qué he dicho blanco si quería decir negro? Técnicas asertivas para el profesorado y formadores. Barcelona: GRAÓ.
Instituto Sonorense de la Mujer. (2014) Manual de trabajo del taller lúdico. Hermosillo, Sonora: Gobierno del Estado de Sonora.

Loaiza de la Pava, J.A., Salazar, M., Santacoloma, J. y León, D. (2015). Seminario lo afectivo en la construcción de la paz. Hermosillo, Sonora: Universidad de Sonora.

Pérez Serrano, G. (coord.). (2011). Intervención sociocomunitaria. Madrid: UNED.

Pérez Serrano, G. y Pérez de Guzmán Puya, M.V. (2011). Aprender a convivir. El conflicto como oportunidad de crecimiento. Madrid: Narcea, S.A. de Ediciones.

Whatling, T. (2013). Mediación: Habilidades y estrategias. Guía Práctica. Madrid: Narcea, S.A. de Ediciones.

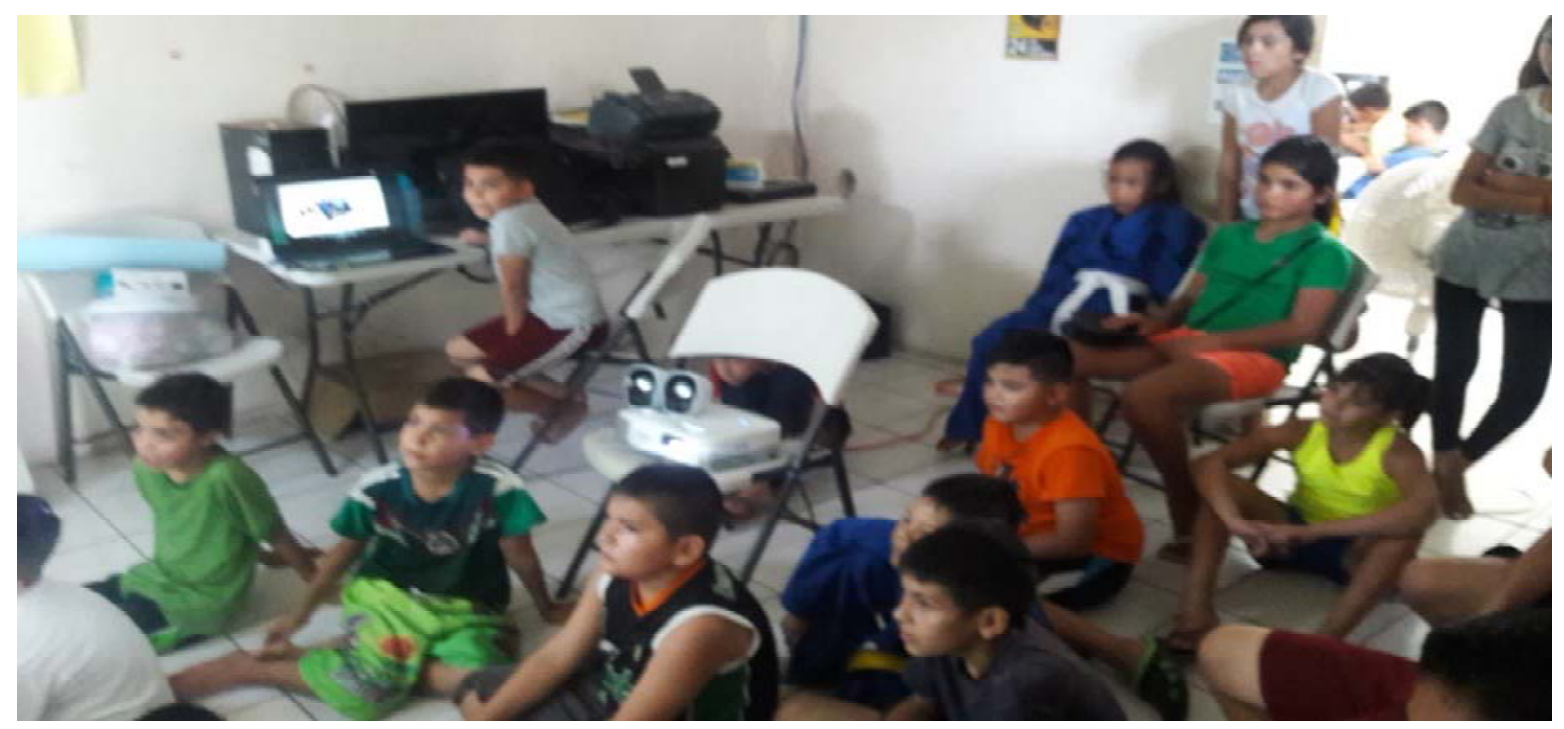

Foto: Patricia Moya Grijalva. Sesión de trabajo 